\title{
Resection of uncovertebral joints and posterior longitudinal ligament for cervical disc arthroplasty
}

\author{
Tsung-Hsi Tu, MD, ${ }^{1-3}$ Chih-Chang Chang, MD, 1,2 Jau-Ching Wu, MD, PhD, ${ }^{1,2}$ Li-Yu Fay, MD, 1,2 \\ Wen-Cheng Huang, MD, PhD, ${ }^{1,2}$ and Henrich Cheng, MD, PhD ${ }^{1,2}$ \\ 1Department of Neurosurgery, Neurological Institute, Taipei Veterans General Hospital; ${ }^{2}$ School of Medicine, National Yang-Ming \\ University; and ${ }^{3}$ Taiwan International Graduate Program in Molecular Medicine, National Yang-Ming University and Academia \\ Sinica, Taipei, Taiwan
}

The most commonly accepted indications for cervical disc arthroplasty (CDA) are 1- and 2-level cervical disc herniation or spondylosis causing radiculopathy or myelopathy that is refractory to medical management. Unlike anterior cervical discectomy and fusion (ACDF), which eliminates motion, CDA aims to restore the physiological range of motion of the indexed joint. Thus, the effect of indirect decompression gained by the insertion of a sufficiently large interbody graft and incorporation into arthrodesis after ACDF cannot be duplicated for CDA. For patients undergoing CDA, during extreme flexion/extension or rotation, the exiting nerve roots might be impinged by inadequately decompressed foraminal osteophytes. Therefore, the authors advocate generous decompression, including resection of the posterior longitudinal ligament (PLL) and bilateral uncovertebral joints (UVJs), even in the asymptomatic side. This video demonstrates full dural expansion and enlarged neuroforamen after removal of the PLL and UVJs. Venous hemorrhage encountered during foraminotomy can always be controlled by cottonoid packing or hemostatic agents. Also, the endplates of the surrounding vertebral bodies were meticulously prepared for parallel insertion of the ProDisc-C Nova (DePuy Synthes Spine) artificial disc. Please note that the ProDisc-C Nova is currently not available on the US market.

The video can be found here: https://youtu.be/XUo34j6WFYs.

KEYWORDS uncovertebral joints; cervical disc arthroplasty; posterior longitudinal ligament; decompression; video 